\title{
Noise Improvement for a High NA MO System
}

\author{
Y. Sabi, Y. Takemoto, K. Aratani, A. Kouchiyama, and A. Nakaoki \\ Giga Byte Laboratories, Sony Corporation, 6-7-35 Kitashinagawa, Shinagawa-ku, Tokyo 141-0001, Japan
}

A feasibility study of a magneto-optical(MO) disk for an NA 0.85 system is presented. The disk noise level in this system was relatively large compared to the conventional NA 0.55 system at the recording density corresponding to this spot size. Two methods were introduced for the improvement. One was the optimization of the groove structure, and the other was the reduction of the metal surface roughness. The former was found to be the typical noise source for optics for a narrower track pitch disk. A UV ashing apparatus was utilized for reduction of the noise. The latter noise source was characteristic for the surface MO recording, and the etching process was found effective for the improvement. By using both these methods, the noise level was drastically decreased, achieving high areal density of $7 \mathrm{~Gb} / \mathrm{inch}^{2}$.

Key words: magneto-optical recording, disk noise, low-pressure $\mathrm{Hg}$ lamp, metal surface roughness

\section{Introduction}

A high NA lens together with a shorter wavelength light source is recognized as the key technology for the next generation optical disk ${ }^{1}$. To show the feasibility of MO disk for such system, we demonstrated a high density MO comprising an NA 0.85 lens $^{2}$. It achieved $10 \mathrm{~GB}$ of capacity for a $12 \mathrm{~cm}$-diameter disk $\left(7 \mathrm{~Gb} /\right.$ inch $\left.^{2}\right)$, by realizing a magnetic field modulation(MFM) recording and read out through a thin cover layer. In this report, among several techniques introduced in this system, we focus mainly on the reduction of MO disk noise, which arose particularly in the high density MO.

Noise due to the narrow track pitch disk was first observed and suppressed. Comparison between NA 0.85 and NA 0.55 systems was carried out using a conventionally sputtered MO film, which is described in section 2 .

To realize a wide skew margin in the high NA system, MO film should be read out through a thin cover or without cover. Accordingly, the film sputtering order was reversed, resulting in a noise increase due to the surface roughness of metal layer. The reduction of this noise is described in section 3 .

Finally, a write and readout experiment was carried out for a high NA surface MO system. The efficiency of the noise reduction methods are shown in section 4 , with a result of high-density recording experiment.

\section{Optimization of the disk groove structure}

As a first step, disk performance was compared between NA 0.85(high NA) and NA 0.55(low NA) systems, at a density proportional to the spot size. The experimental conditions for each of these systems are shown in Table 1. For both systems, a conventional TbFeCo film was sputtered so that the laser beam was introduced from the substrate side, and recording was made using MFM with a conventional flying head.

Table 2 is the result of jitter and $\mathrm{C} / \mathrm{N}$, and corresponding noise spectra are shown in Fig. 1a and $1 \mathrm{~b}$. The high NA system showed larger erase noise resulting in a higher jitter level. From the comparison of noise levels between groove and mirror areas, it was apparent that the noise was mostly originated from the groove boundary wall. The groove boundary size and shape is determined by the optics and the photoresist used in the mastering process. As the track pitch becomes narrower, the boundary area occupies relatively larger ratio to the recording area, and this may lead to the higher noise level in the high NA

Table 1. Experimental condition of each system.

\begin{tabular}{c|c|c|c|c} 
& NA & Wavelength & $\begin{array}{c}\text { Substrate } \\
\text { thickness }\end{array}$ & $\begin{array}{c}\text { Linear } \\
\text { velocity }\end{array}$ \\
\hline Low NA & 0.55 & $690 \mathrm{~nm}$ & $1.2 \mathrm{~mm}$ & $4.6 \mathrm{~m} / \mathrm{s}$ \\
\hline High NA & 0.85 & $635 \mathrm{~nm}$ & $0.6 \mathrm{~mm}$ & $2.8 \mathrm{~m} / \mathrm{s}$ \\
\hline
\end{tabular}

Table 2. Recording characteristics of each disk.

\begin{tabular}{c|c|c|c|c|c}
\hline Disk & $\begin{array}{c}\text { Track } \\
\text { pitch } \\
(\mu \mathrm{m})\end{array}$ & $\begin{array}{c}\text { Bit } \\
\text { length } \\
(\mu \mathrm{m})\end{array}$ & $\begin{array}{c}\text { Jitter } \\
(\%)\end{array}$ & $\begin{array}{c}\mathrm{C} / \mathrm{N}^{*} \\
(\mathrm{~dB})\end{array}$ & $\begin{array}{c}\text { Noise } \\
\text { level } \\
(\mathrm{dBm})\end{array}$ \\
\hline Low NA & 0.85 & 0.35 & 7.2 & 56.5 & -61.8 \\
\hline High NA & 0.55 & 0.21 & 9.0 & 50.5 & -57.1 \\
\hline $\begin{array}{l}\text { High NA } \\
\text { improved }\end{array}$ & 0.55 & 0.21 & 7.3 & 55.2 & -60.6 \\
\hline
\end{tabular}

*Mark length: $1.87 \mu \mathrm{m}$ for low NA, and $1.12 \mu \mathrm{m}$ for high NA. 


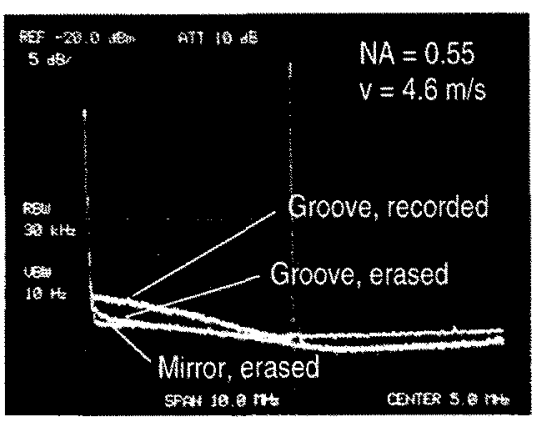

Fig. 1a Noise spectrum of a low NA system.

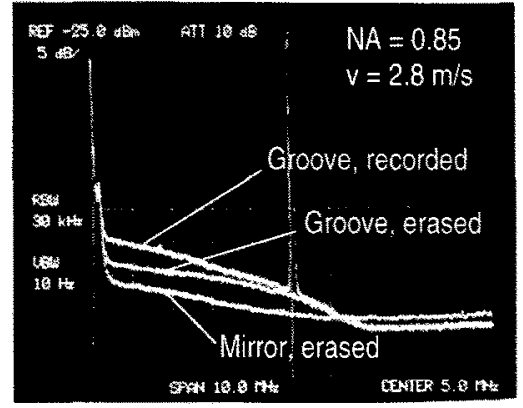

Fig. 1b Noise spectrum of a high NA system.

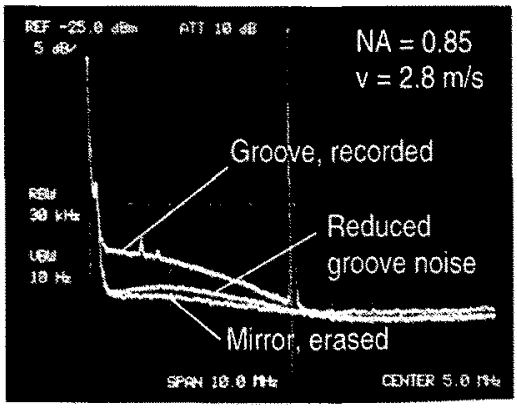

Fig. 1c Noise spectrum of a high NA system for an improved substrate.

system.

To reduce the groove noise, the groove surface was improved using a $\mathrm{UV} / \mathrm{O}_{3}$ cleaner (ORC Co., PES-730 prototype). After the irradiation by lowpressure mercury lamp of $0.12 \mathrm{~J} / \mathrm{cm}^{2} \cdot \min$ for 10 minutes, the surface roughness and the groove edge curvature was reduced, as can be clearly seen in the cross sectional image of the substrate by an SEM (Fig. 2). An identical wide groove glass $2 \mathrm{P}$ (photo-polymerization) substrate was used in this experiment, through the following sections.

As a result, the noise level decreased drastically as shown in Fig. 1c, in which the noise profile was measured at the same condition as in Fig. 1b. The noise level decreased both for the mirror and the groove area; however, drastic progress was made for the latter. The $\mathrm{C} / \mathrm{N}$ and jitter also improved as shown in Table 2. The improvement of noise can be explained by the lower fluctuation of magnetic properties at the smoother boundary wall.

A $\mathrm{UV} / \mathrm{O}_{3}$ cleaner is a popular apparatus for removing the photoresist, with its mechanism well known. The effect introduced in this experiment is considered simply the same. The surface of photopolymer was gradually removed by the excited oxygen atom that was produced by the UV light, so the groove depth was reduced by the screening effect. Accordingly, the pull-in level, namely the reflected light intensity from the groove, is increased due to the widened recording area, and the tracking error signal level is decreased due to the reduced groove depth(Fig. 3 ).

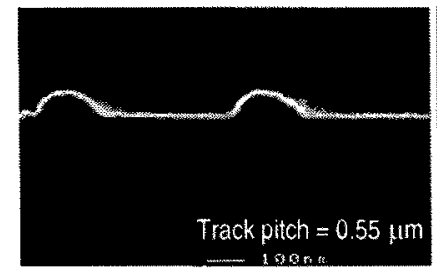

Fig. 2a An SEM image of a wide groove substrate.

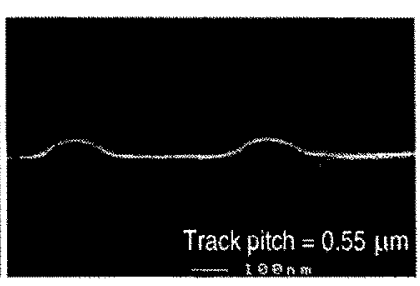

Fig. 2b An SEM image of an improved substrate.
To find the optimum UV irradiation time taking into account the cross writing from the adjacent tracks, the jitter was measured after recording on two adjacent tracks. As shown in Fig. 4, the jitter was improved as the UV irradiation time increased while keeping the write power margin wide. Within the measured range, the processed groove structure prevented the domain wall from moving to the adjacent track during the recording. Thus the servo signal level determined the limitation on the UV irradiation time. To obtain a sufficient error signal level, we chose 10 minutes of irradiation time.

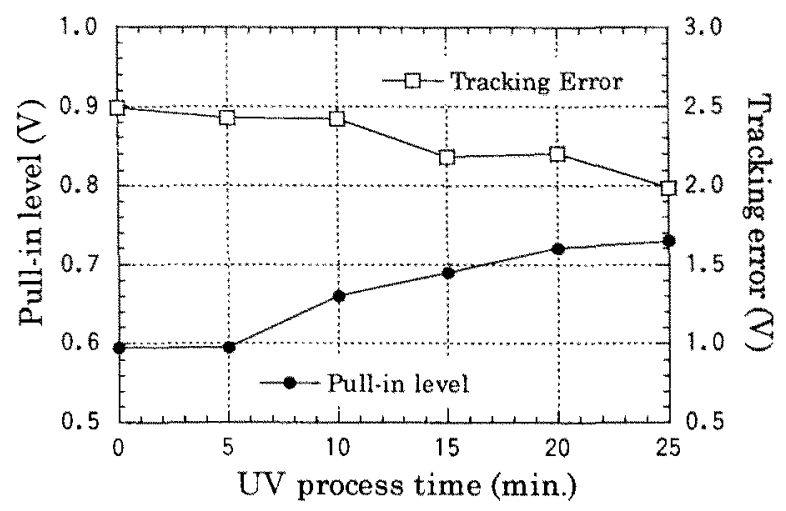

Fig. 3 Pull-in level and tracking error level change as a function of UV irradiation time.

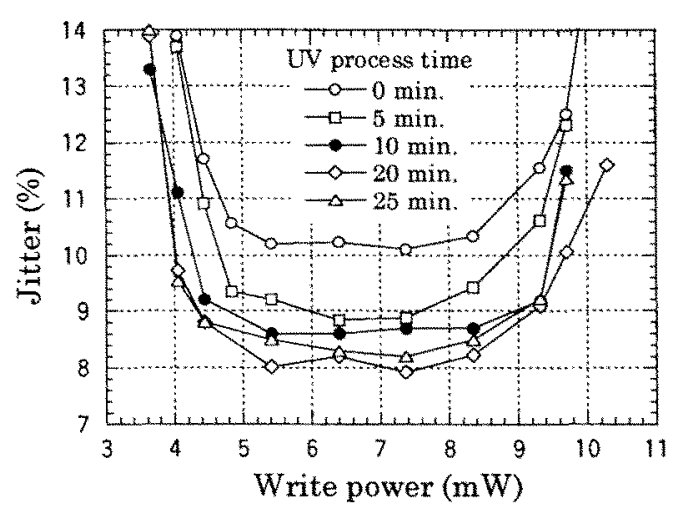

Fig. 4 Jitter level of improved substrates. 

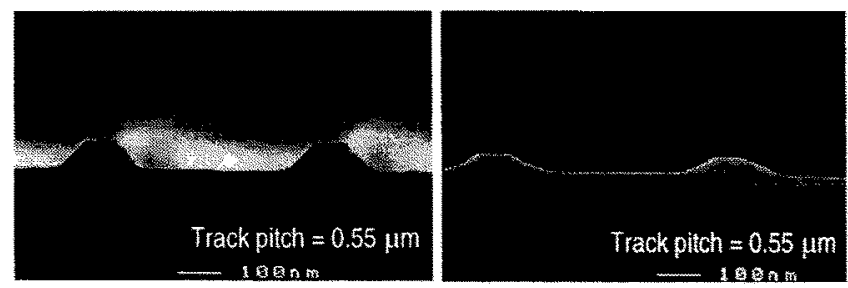

Fig. 5a A SEM image of a PC substrate.

Fig. 5b A SEM image of an improved $\mathrm{PC}$ substrate.

A similar result was obtained for a polycarbonate(PC) substrate. Since the ashing effect was faster for the PC material, a few minutes of the UV irradiation time was sufficient to obtain the same result. The SEM images of the PC substrate are shown in Fig. 5.

This method was also valid for the low NA system; however, the effect was relatively small compared to the high NA system. This result can be expected from the well-optimized noise profile in Fig. 1a. The UV irradiation was considered more effective for the narrow track pitch disks.

\section{Reducing the metal surface roughness}

Another requirement for the high NA system was a thin transparent substrate, namely a cover, which was inevitable to realize a wide skew margin. Accordingly, when using the conventional $\mathrm{TbFeCo}$ film, the sputtering process was reversed such that a reflective layer was sputtered as an underlayer of the MO film(Fig. 7). Compared to the $\mathrm{TbFeCo}$ or the SiN protective layer, the surface of the reflective layer tends to be rough due to the growth of crystal particles. Thus the rough surface of the metal film increases the noise level both on the groove and at the groove boundary.

The surface roughness, especially for the groove boundary, strongly depends on the material and its sputtering condition. Furthermore, a relation between the surface roughness and the noise level did depend on the optical system; in the high NA system, the higher optical resolution led to a stronger dependence of the noise upon the roughness.

On the optimization of the material and the sputtering condition, erased noise level itself was used as a parameter since it was difficult to characterize the roughness at the groove boundary. After the optimization, a Ag based material was chosen. However, the noise level did not reach the level as low as the normally processed film(e.g. Fig. $1 b$ or $1 c)$, in which the surface roughness does not

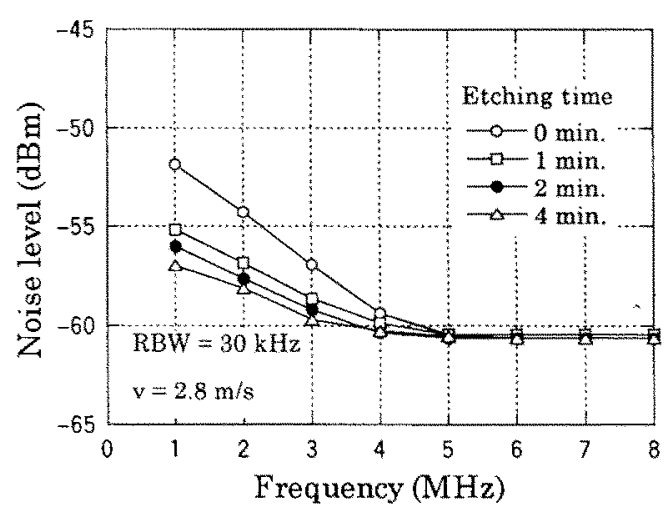

Fig. 6a Erase noise level on flat surface.

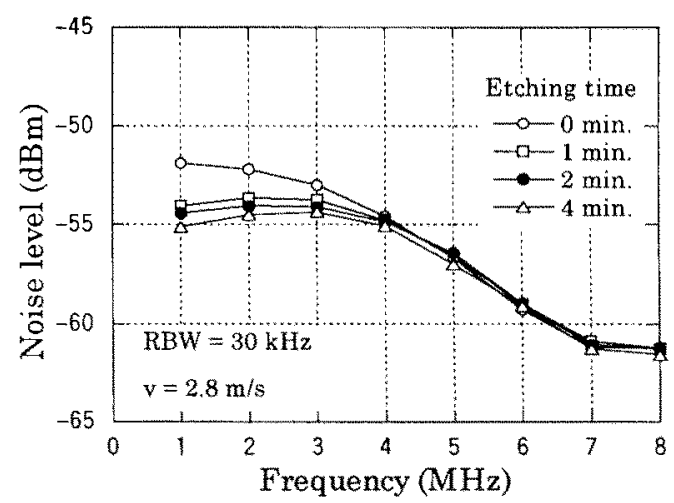

Fig. 6b Erase noise level on groove.

affect the noise level.

To reduce the noise, the metal layer was sputter etched to smooth the surface. Figure $6 a$ and $6 b$ show the noise spectrum for different sputter etching times, with $\mathrm{TbFeCo}$ film subsequently sputtered and erased. The etching power was $50 \mathrm{~W}$ and the background Ar pressure was $0.30 \mathrm{~Pa}$. For both on the mirror surface and on the groove, the noise level was reduced according to the etching time. By the etching time of 4 minutes, the noise level decreased well below to the same level as the normally processed film, as can be seen from the comparison with Fig. $1 \mathrm{~b}$.

By measuring with STM, it was found that Ra value of the flat surface area decreased from 1.1 $\mathrm{nm}$ to $0.60 \mathrm{~nm}$ by the etching process. The noise level of the flat surface was also measured in the low NA system, yielded no difference in the range of etching time mentioned above. The difference was observed only for the high resolution optics.

\section{High NA record/readout experiment}

To confirm the performance of the improved low noise media, an MFM-high NA optics was adopted as shown in Fig. 7. A 2-element lens of NA 0.85 focused a laser light of $635 \mathrm{~nm}$ on the MO film 


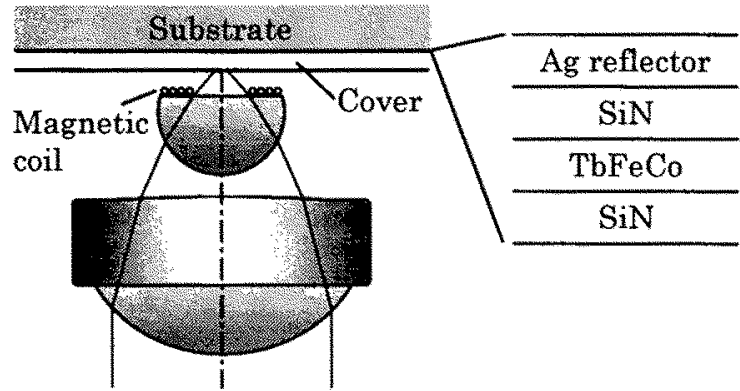

Fig. 7 Schematic diagram of the high NA pick up and the magneto-optical disk.

through the center hole of a thin film coil. The distance between the MO film and the coil was set to $50 \mu \mathrm{m}$ in order to produce the magnetic field of above 150 Oe on the film. The design parameters of the optical system such as the cover thickness or the coil diameter is yet to be investigated, however, its optical performance was tuned enough to analyze the MO disk.

Figure 8 is the $\mathrm{C} / \mathrm{N}$ profile of the disk with and without the improvement technique described above, and Fig. 9 is the erase noise level on the groove for each of the disks. Both the UV irradiation and the metal etching were proved to be effective in this system.

Comparing with the conventional system(Table

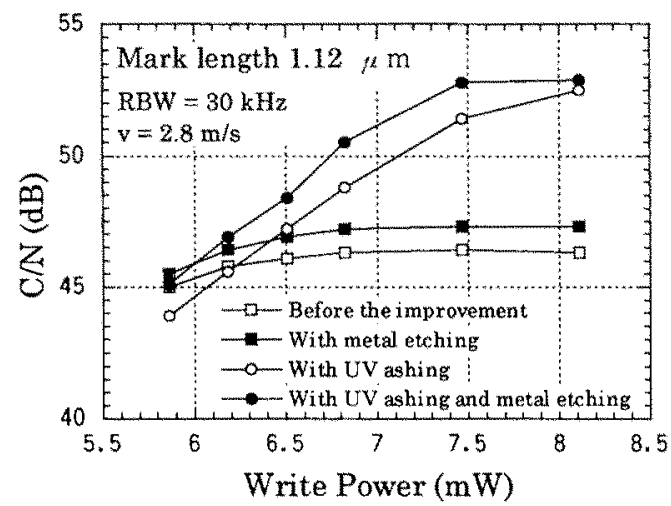

Fig. $8 \mathrm{C} / \mathrm{N}$ of the improved and unimproved disks.

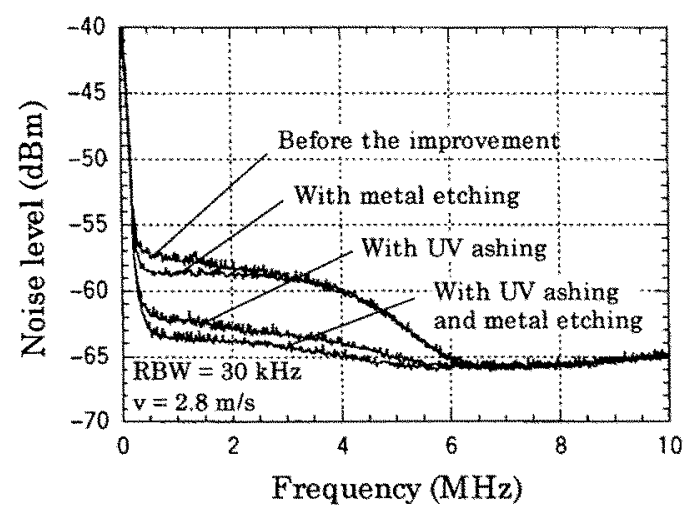

Fig. 9 Erase noise profile of each disk on groove.
2 ), the maximum $\mathrm{C} / \mathrm{N}$ was still lower. Since the difference mostly occurred from the write noise level, this is explained by the insufficient magnetic field sensitivity of the disk. Further optimization of the MO film or production of larger magnetic field will lead to a better result.

Under these conditions, the $(1,7)$ modulated random data was recorded under a channel clock of $20 \mathrm{MHz}$. With the benefit of PRML(1,2,1), a low error rate of less than $10^{-4}$ was obtained at the bit length of $0.17 \mu \mathrm{m} / \mathrm{bit}$, which corresponds to the high areal density of $7 \mathrm{Gbit} / \mathrm{inch}^{2}$ (Fig.10).

With the improvement of the noise level, MO disk exhibited high performance in a high NA recording. This result promises that further optimization as a whole system will lead to higher density recording.

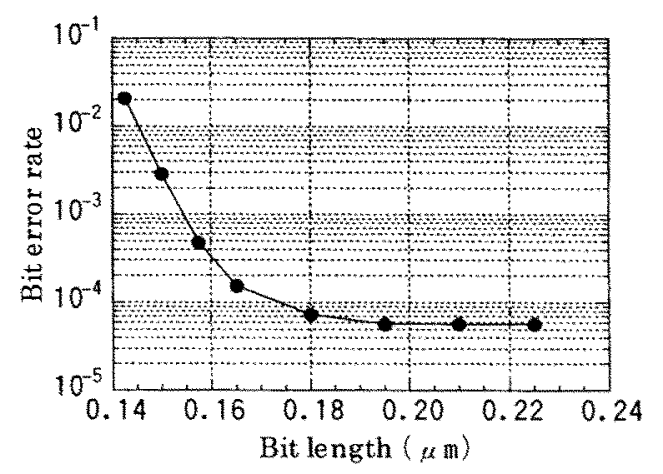

Fig. 10 Bit error rate of the NA 0.85 MO system.

\section{Conclusion}

With a high NA MO system, noise level increased due to the narrower track pitch and to the reversed sputtering process. The media noise originated mostly from the groove boundary, which was observed to be significant for the high NA. By the surface improvement of the substrate and the reflective layer, the S/N drastically improved.

These results suggest that the MO medium with these techniques should be advantageous even at the higher density MO realized by the shorter wavelength or the near field optics.

\section{References}

1) K. Osato, K. Yamamoto, I. Ichimura, F. Maeda, and Y. Kasami, Digest of Optical Data Storage (Aspen, Colorado, 1998), p. 131

2) A press release by SonyCorporation, October 22, 1997, Tokyo, http://www.sony.co.jp/soj/CorporateInfo/News/1 99710/97-099/index.html (in Japanese only) 